\title{
On The Use of Methylimidazolium Acetate Ionic Liquids as Solvent and Stabilizer in the Synthesis of Cobalt Nanoparticles by Chemical Reduction Method
}

\author{
Walter Efiok John, Ayi Anyama Ayi (i) *, Chinyere Ayi Anyama, Providence Betelwhobel \\ Ashishie, Bassey Enyi Inah
}

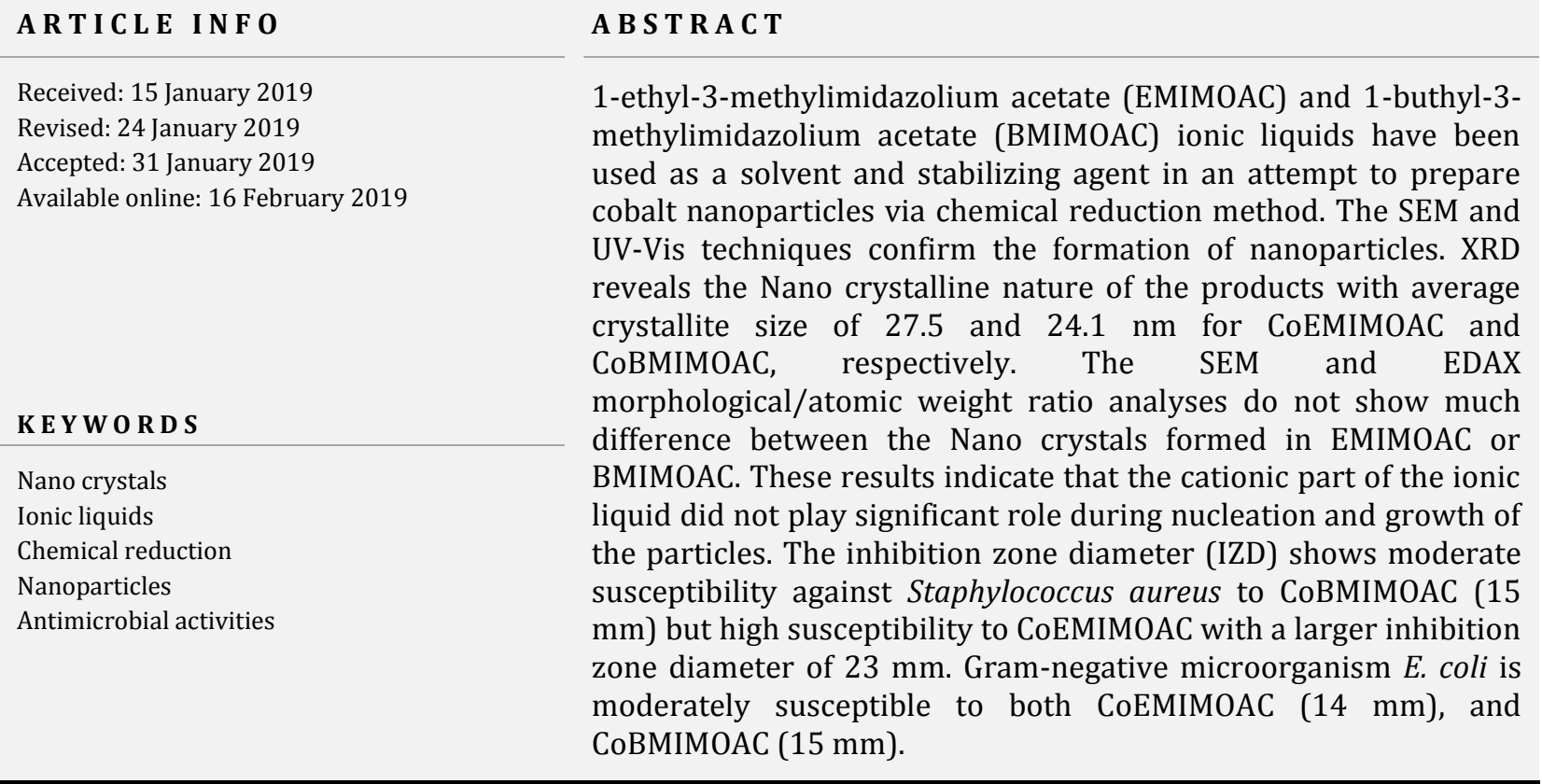

G RA P H I C A L A B S T RACT

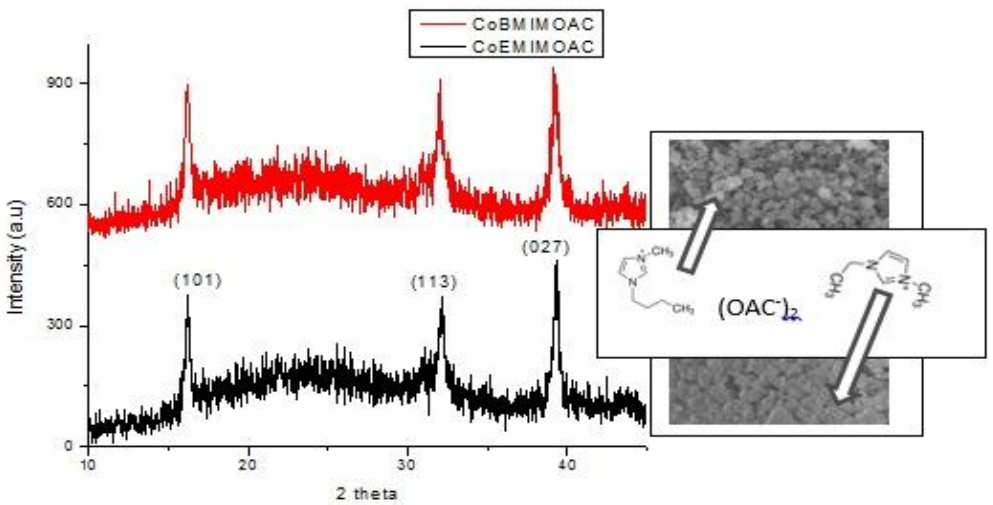

* Corresponding author's E-mail address: ayiayi72@gmail.com, a.anyama@unical.edu.ng, Tel.: $+2348035961521$

Inorganic Materials Research Laboratory, Department of Pure and Applied Chemistry, University of Calabar, Nigeria. 


\section{Introduction}

The use of ionic liquids (ILs) in the synthesis of inorganic nanomaterials has received great attention [1-7], not only for being environmentally benign solvent, but also for ease of preparing complex inorganic materials that cannot be made using conventional solvents [8-11]. Ionic liquids (ILs) can stabilize metal and metal oxide nanoparticles through their high ionic charge, their polarity and high dielectric constants [12-16].

The physical size of materials can be used to tune materials properties. Properties such as optical, magnetic, and catalytic properties are quite interesting at the nanometer size regime with resultant applications in chemical technology, magnetic data storage, and sensing, etc. [17]. Efforts to explore structures on the nanometer length scale unite the frontiers of materials chemistry, physics, and engineering. It is in the design and characterization of advanced materials that the importance of new interdisciplinary studies may be realized. Uncovering and mapping size-dependent materials properties requires synthetic routes to prepare homologous size series of monodisperse nanometer size crystals, known as nanocrystals (NCs). NC samples must be monodisperse in terms of size, shape, internal structure, and surface chemistry [18]. In the study, ionic liquids based on 1-ethyl-3-methylimidazolium (EMIM) or 1-butyl-3-methylimidazolium (BMIM) cations and acetate anion have been used to prepare cobalt-based Nano crystals and their antibacterial properties evaluated.

\section{Materials and methods}

\section{Materials}

Cobalt (II) chloride hexahydrate $\left(\mathrm{CoCl}_{2} \cdot 6 \mathrm{H}_{2} \mathrm{O}\right)$, Sodium tetrahydroborate (III) $\left[\mathrm{NaBH}_{4}\right]$, Sodium hydroxide, ionic liquid based on 1-ethyl-3-methylimidazolium (EMIM) and 1-buthyl-3-methylimidazolium (BMIM) cations and acetate anions (OAc) were obtained from commercial source and used as received.

\section{Preparation of the Cobalt Nano crystals}

Exactly $23.793 \mathrm{~g}$ of cobalt (II) chloride hexahydrate (assay $=99.9 \%$ ) was dissolved in $50 \mathrm{~cm}^{3}$ of ethylene glycol (ethane-1, 2diol). A portion of this solution $\left(8 \mathrm{~cm}^{3}\right)$ was dispersed in $3 \mathrm{~cm}^{3}$ of 1-ethyl-3methylimidazolium acetate (EMIMOAC)/or 1-buthyl-3-methylimidazolium acetate (BMIMOAC) under constant stirring at room temperature. The maroon-blue solution became black on addition of $\mathrm{NaBH}_{4}(0.00945$

g) and $1.5 \mathrm{~cm}^{3}$ of $0.5 \mathrm{M} \mathrm{NaOH}$ with evolution of colourless fumes. The mixture was then heated to $70{ }^{\circ} \mathrm{C}$ within $3 \mathrm{~h}$. The colloidal dispersion obtained was centrifuged and the 
precipitate washed and dried at room temperature.

\section{Antimicrobial Studies}

Antimicrobial test was carried out in the Department of Microbiology, University of Calabar. The disc diffusion method was adopted [19]. With the help of a cotton swap, isolated colonies of the organisms were suspended in $5 \mathrm{~cm}^{3}$ saline Mueller-Hilton. The turbidity of the suspension was matched with the turbidity standard (equivalent to a 0.5 McFarland standard). A discrete colony of each of the isolate was picked with a sterile wire loop and streaked on the Muller-Hilton agar plate. About 2000 $\mu \mathrm{g}$ of CoEMIMOAC, CoBMIMOAC and amoxicillin were transferred to the agar plates. Within 30 minutes of applying the disc, the plate was inverted and incubated aerobically at $35{ }^{\circ} \mathrm{C}$ for $18 \mathrm{~h}$. At the end of incubation, zones of inhibition (ZOI) were measured and recorded in millimeters.

\section{Characterization}

The samples were analyzed by Rigaku MiniFlex II X-ray diffractometer using monochromatic $\mathrm{Cu}$ K $\alpha$ radiation $(\lambda=0.1541$ $\mathrm{nm}$ ) at the speed of $3 \mathrm{~s}$ in $2 \theta$ range between 5 - 750 and step size of 0.03 .

The peaks were analyzed using the Scherrer formula [20]:

$$
D_{h k l}=\frac{k \lambda}{\beta\left(\frac{\pi}{180}\right) \cos \theta}
$$

Where $D_{h k l}$ is the coherence length of the crystalline domain perpendicular to the respective $h k l$ plane, $\mathrm{k}$ is a constant (here $0.9), \lambda$ is the wavelength of $\mathrm{CuK \alpha}$ radiation (1.5406 $\AA$ ), $\beta$ is the background corrected line broadening in degrees, $(\pi / 180)$ is a correction factor to calculate $\beta$ in radians, and $\theta$ is the scattering angle. The scanning electron microscopy (SEM) studies of the nanocrystals were made with a Field Emission Electron Microscope (FESEM JSM$6700 \mathrm{~F}$ ), coupled with an energy dispersion analyzer (EDX). The specimens were $\mathrm{Au}$ coated (sputtering) to make them conductive. The SEM acceleration voltage was $10 \mathrm{kV}$. The Fourier transform infrared (FTIR) spectra for the synthesized Nano crystals were recorded over the wave number range of $400-4000 \mathrm{~cm}^{-1}$ using Perkin Elmer FTIR spectrometer. The powdered samples were mixed with $\mathrm{KBr}$ (in a 1: 200 ratio of their weight) and pressed in the form of pellets for measurement. Ultraviolet/Visible Spectroscopic measurements were carried out using 201 Evolution UV-Visible spectrophotometer. A portion $\left(1 \mathrm{~cm}^{3}\right)$ of methanolic solution of the precipitates $\left(0.1 \mathrm{~g}\right.$ of solid dissolved in $5 \mathrm{~cm}^{3}$ of methanol) was taken out and dispersed in $5 \mathrm{~cm}^{3}$ of distilled water for the UV-Vis measurement.

\section{Results and discussion}

Nano crystals have been synthesized via 
chemical reduction of the cobalt chloride elevated temperatures to obtain homogeneous hexahydrate by $\mathrm{NaBH} 4$ in ILs EMIMOAC / or reaction mixtures. The colour changes from BMIMOAC. The reactions were performed at maroon blue to black.

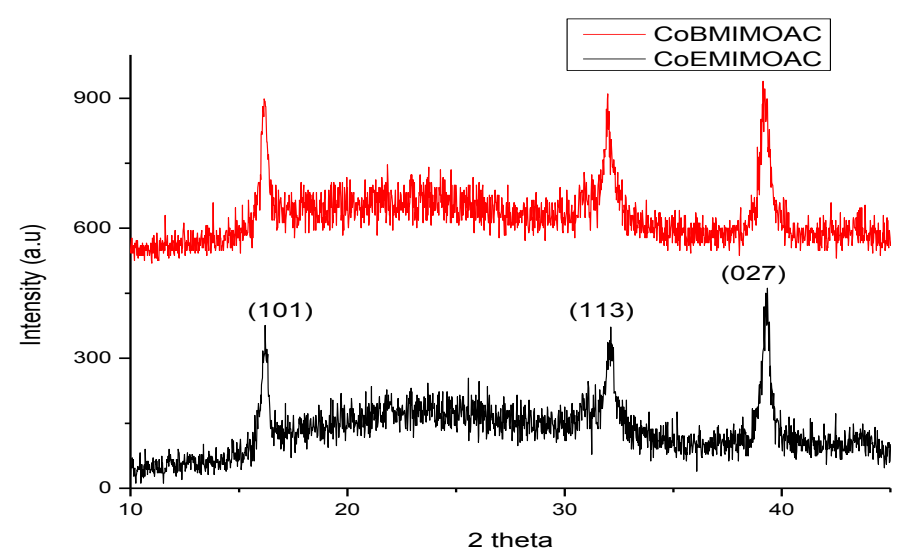

Figure 1. Powder X-ray diffraction pattern of the nanocrystals

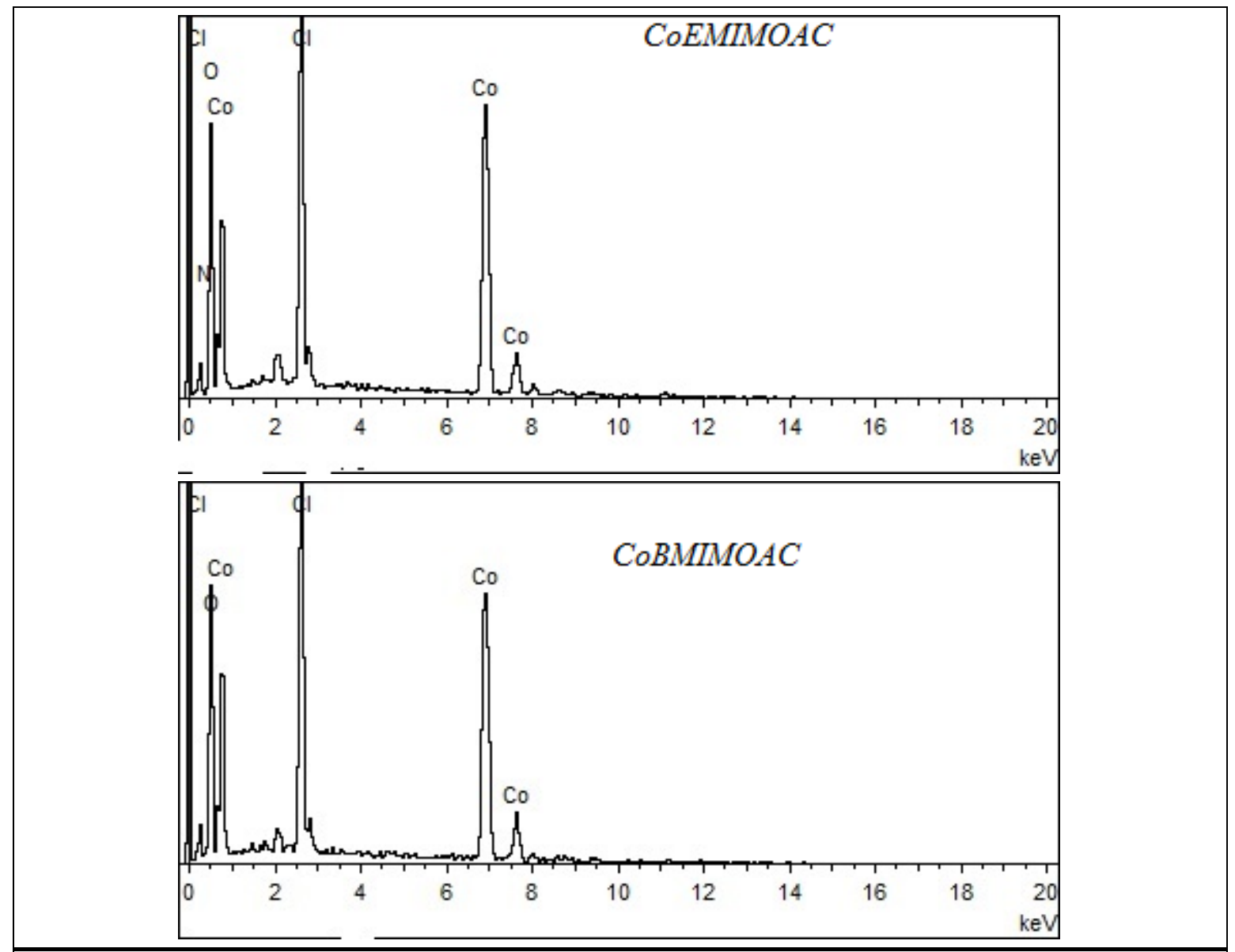

Figure 2. EDAX spectra of as-synthesized cobalt nanocrystals grown in imidazolium-based ILs 
John et al.

Figure 1 shows a typical X-ray diffraction (XRD) pattern of the nanocrystals formed after isolation and drying. XRD identifies the products to be Cobalt hydroxide chloride (Ref. code $=01-073-2134$, ISCD $=24685)$. The most representative reflections of $\mathrm{Co}_{2}(\mathrm{OH})_{3} \mathrm{Cl}$ were indexed in rhombohedral crystal space group R-3m (No.166) with the following unit cell dimensions $\mathrm{a}=\mathrm{a}=6.8400 \AA, \mathrm{c}=14.500 \AA, \alpha$ $=\beta=90^{\circ}, \gamma=120^{\circ}$, Vol. $=587.50 \AA^{3}, Z=6$. The representative reflections of (101), (113), and (024) were observed at $2 \theta$ values of $16.2,32.12$ and $39.26^{\circ}$, respectively with corresponding interplanar distances of $d_{101}=$ 5.4837, $d_{113}=2.7918$ and $d_{024}=$ $2.2936 \AA$. The average crystallite size $D_{h k l}$ (coherence lengths) calculated using Scherrer equation [19] is $27.5 \mathrm{~nm}$ for CoEMIMOAC and $24.1 \mathrm{~nm}$ for CoBMIMOAC. For the XRD pattern shown in Figure 1 , the $D_{101}$ is $29.13, D_{113}$ is $26.26, D_{024}$ is 35.72 , and $D_{027}$ is $18.85 \mathrm{~nm}$.

Figure 2 shows the energy dispersive analysis by X-ray (EDAX), while Figure 3 gives representative scanning electron microscopy (SEM) images of the products. The EDAX results for sample grown in EMIMOAC ionic liquid showed the percentage of cobalt, chlorine, oxygen and nitrogen to be 50.66, $16.19,31.51$ and $1.64 \%$, respectively. Whereas the percentage of cobalt, chlorine and oxygen is 49.16, 17.25 and $33.59 \%$, respectively for sample grown in BMIMOAC. The SEM reveals a monodisperse Nano crystals in both EMIMOAC and BMIMOAC.

Figure 4 shows the UV-Vis spectrum of sample grown in EMIMOAC. The spectrum exhibit well-defined absorption bands which can be assigned to the surface Plasmon absorption of the Nano crystals. The surface Plasmon resonance can be thought of as the coherent motion of the conduction-band electrons caused by interaction with an electromagnetic field. The frequency and width of the surface Plasmon absorption depend on the size and shape of the metal nanoparticle as well as on the dielectric constant of the metal itself and of the medium surrounding it [21-23]. It can be seen in Figure 4 that the surface Plasmon resonance splits into two bands, with the higher energy band in the range 430-460 nm and the lower energy in the region $500-560$ nm.

FTIR spectrum of the Nano crystals grown in either EMIMOAC or BMIMOAC ionic liquid (Figure 5) showed broad absorption band centered around $3447 \mathrm{~cm}^{-1}$, which can be attributed to the N-H stretching vibration. The bands at 1504 and $1410 \mathrm{~cm}^{-1}$ can be assigned to $\mathrm{C}=\mathrm{C}$ vibrations of the imidazolium ring indicating that the ionic liquids have been adsorbed on the surface of the nanocrystals thus providing electrosteric stabilization of the particles in agreement with literature reports [24-30]. The bands in the region 656-426 $\mathrm{cm}^{-1}$ are attributed to Co-O vibrations. 


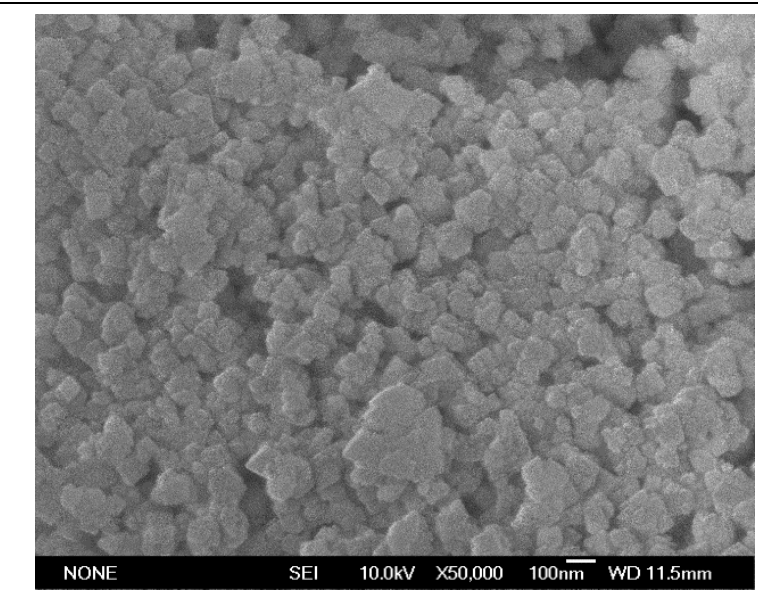

(a)

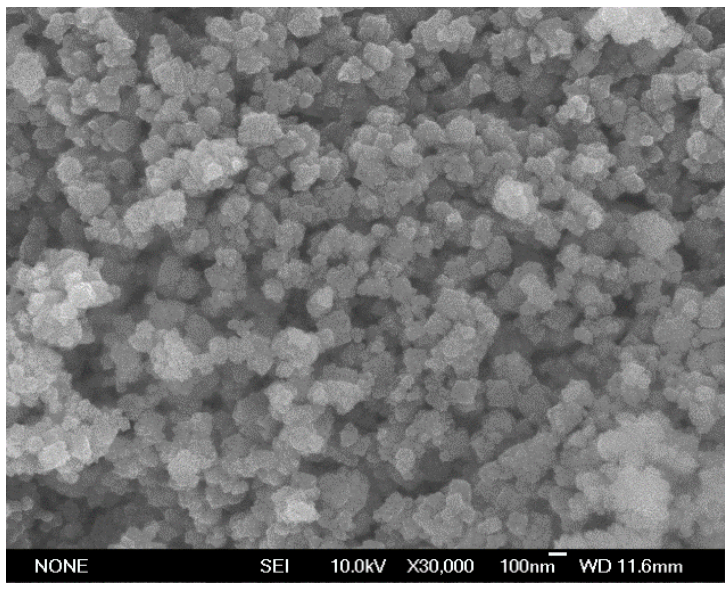

(b)

Figure 3. Representative SEM images of particles grown in (a) EMIMOAC (b) BMIMOAC

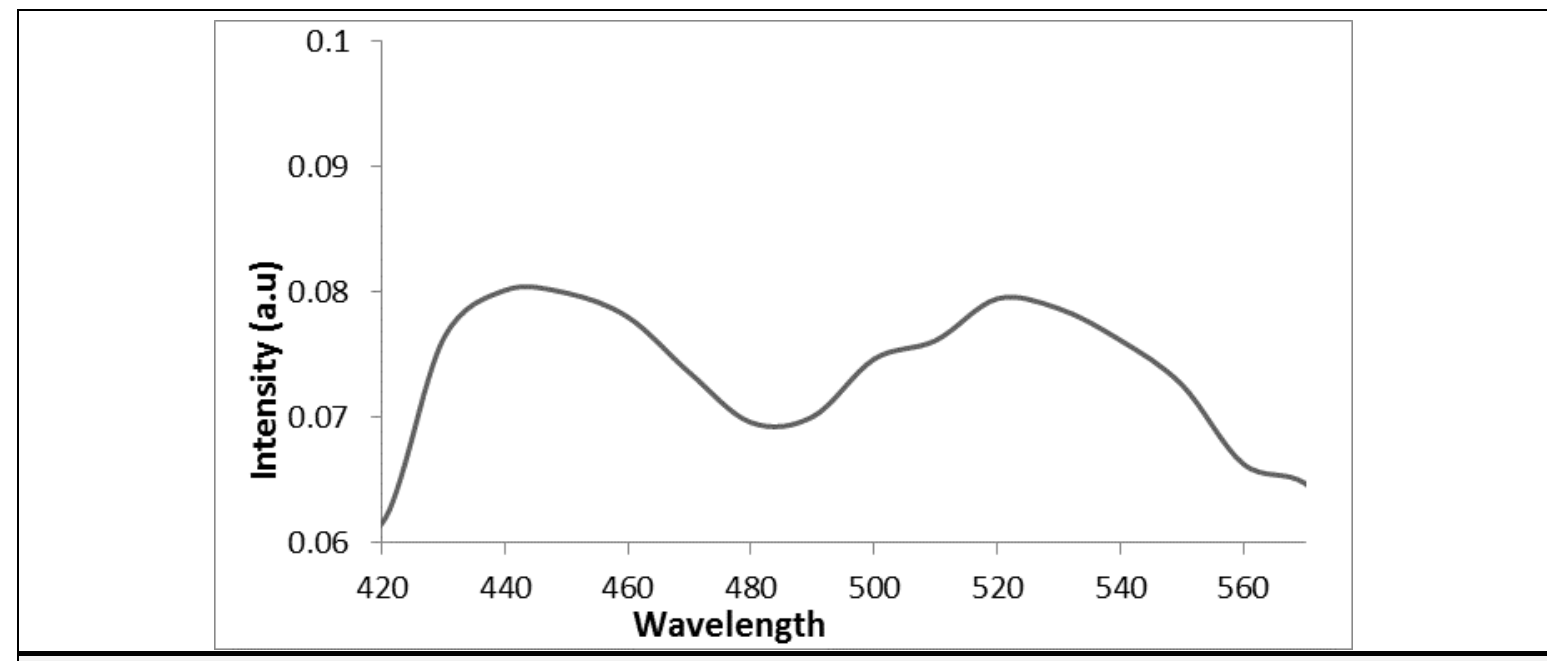

Figure 4. UV-Visible spectrum of cobalt nanoparticles in IL EMIMOAC

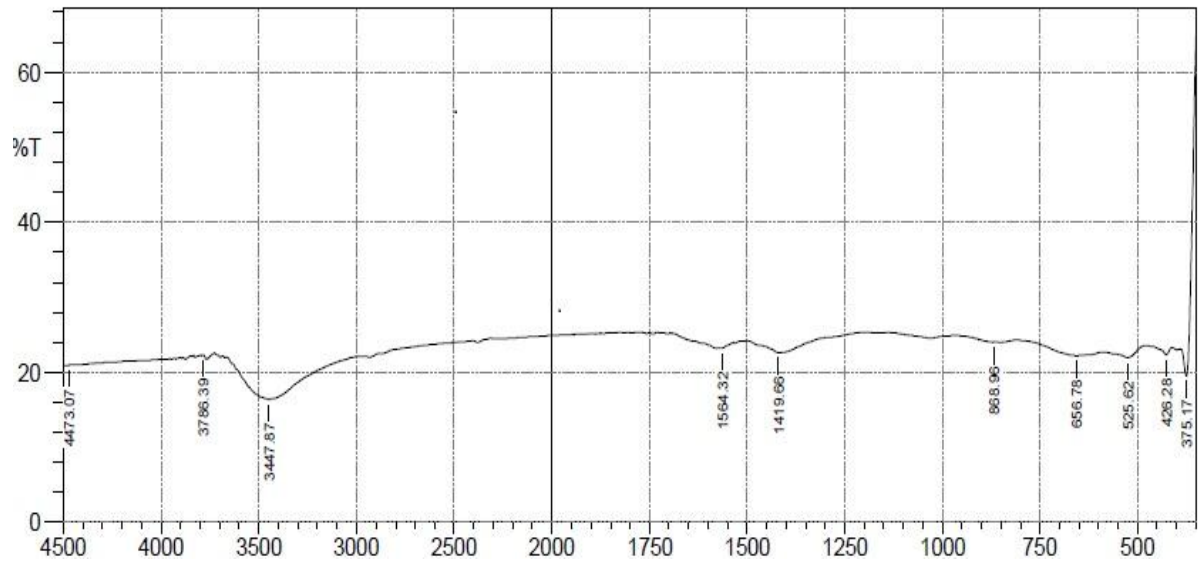

Figure 5. FTIR spectrum of particles grown in EMIMOAC

180 Adv J Chem A 2019, 2(2), 175-183| http://ajchem-a.com 
The potential of the synthesized Nano crystals to inhibit the bacterial growth was evaluated and comparison made to the over the counter drug amoxicillin as shown in Table 1, Figure 6. The inhibition zone diameter (IZD) shows moderate susceptibility against Staphylococcus aureus to CoBMIMOAC (15 mm) but high susceptibility to CoEMIMOAC with a larger inhibition zone diameter of $23 \mathrm{~mm}$. Gramnegative microorganism E. coli is moderately susceptible to both CoEMIMOAC (14 mm), and CoBMIMOAC (15 mm). The inhibition zone diameter of CoEMIMOAC show high susceptibility to Staphylococcus aureus and moderately susceptible to $E$. coli suggesting its high susceptibility to gram-positive microorganism than gram-negative (E. coli). The Nano drug: CoEMIMOAC performs better than amoxicillin in inhibiting of Staphylococcus aureus.

Table 1. Zones of inhibition (ZOI) of bacterial growth by the synthesized nanocrystals

\begin{tabular}{cccc}
\hline & CoEMIMOAC & CoBMIMOAC & Amoxicillin \\
\hline & ZOI & ZOI & ZOI \\
Staphylococcus aureus & $23 \mathrm{~mm}$ & $15 \mathrm{~mm}$ & $14 \mathrm{~mm}$ \\
Escherichia coli & $14 \mathrm{~mm}$ & $15 \mathrm{~mm}$ & $20 \mathrm{~mm}$ \\
\hline
\end{tabular}
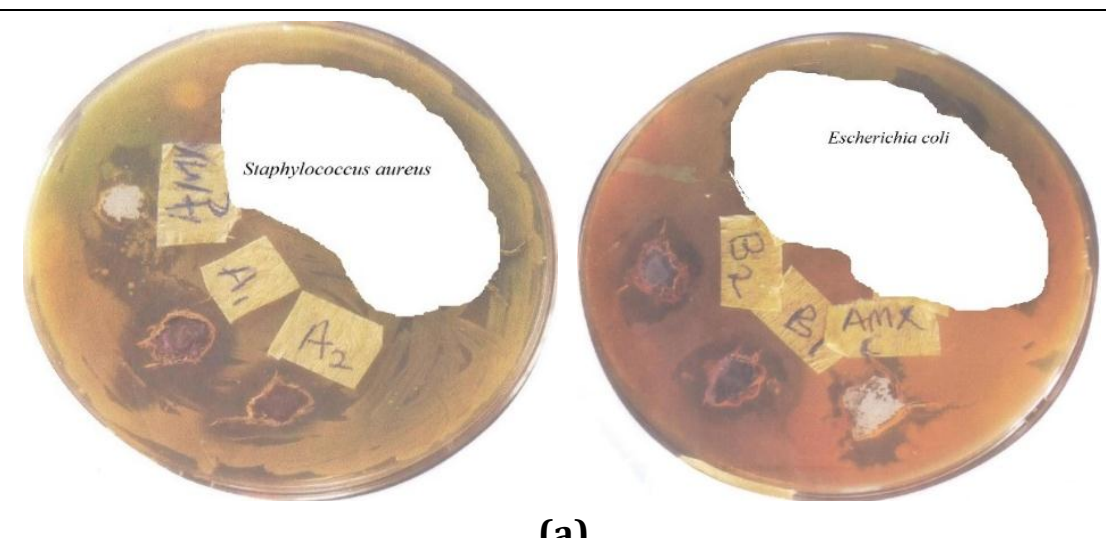

(a)

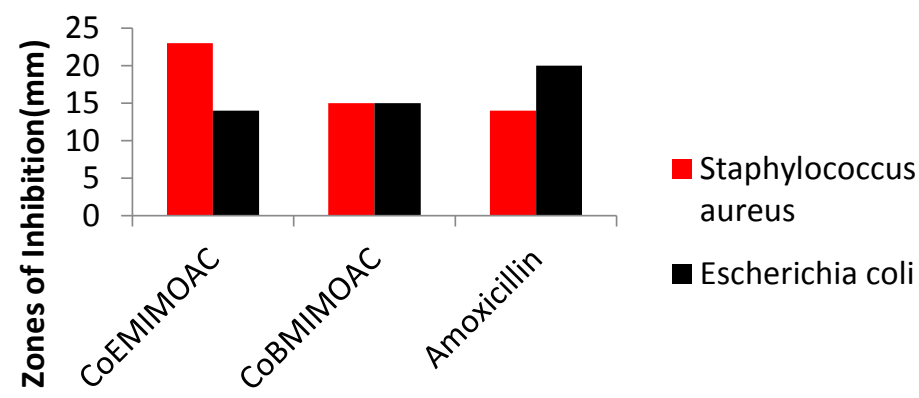

Nanostructures and antibiotics

Figure 6. (a) Agar plates showing zones of inhibition. Staphylococcus aureus: CoEMIMOAC $=\mathrm{A}_{1}$ and CoBMIMOAC $=\mathrm{A}_{2} ;$ Escherichia coli: $\mathrm{CoEMIMOAC}=\mathrm{B}_{1}$ and $\mathrm{CoBMIMOAC}=\mathrm{B}_{2}$, Amoxicillin $=\mathrm{AMX}$

(b) Bar chart showing inhibition zone diameter 


\section{Conclusion}

In conclusion, 1-ethyl-3-methylimidazolium acetate (EMIMOAC)/or 1-buthyl-3methylimidazolium acetate (BMIMOAC) ionic liquids have been successfully used as a solvent and stabilizer in the synthesis of Nano crystals of cobalt. The SEM and UV-Vis techniques confirm the formation of nanoparticles. XRD revealed the Nano crystalline nature of the products. There was no difference between the Nano crystals formed in EMIMOAC or BMIMOAC indicating that the cationic part of the ionic liquid did not play significant role during nucleation and growth of the particles. The zone of inhibition of CoEMIMOAC obtained against $S$. aureus and E. coli are 23 and $14 \mathrm{~mm}$, respectively whereas that of CoBMIMOAC against the two bacterial under investigation is $15 \mathrm{~mm}$. The synthesized Nano crystals have therefore demonstrated great potentials as Nano drugs in the treatment of infections caused by gram-positive microorganisms.

\section{Acknowledgement}

This work was supported by The World Academy of Sciences for the Advancement of Science in developing countries (TWAS) under Research Grant No 12-169 RG/CHE/AF/AC-G UNESCO FR: 3240271320 for which grateful acknowledgment is made. A.A.A is also grateful to the Royal Society of Chemistry for personal Research grant. The assistance of Professor J -G. Mao of Fujian Institute of Research on the
Structure of Matter, Chinese Academy of Sciences, in the XRD and SEM analyses is gratefully acknowledged.

\section{ORCID}

Ayi Anyama Ayi (D: 0000-0002-4908-9929

\section{References}

[1]. P.B. Ashishie, B.E. Inah, A.A. Ayi. Int. J. Sci., 2018, 7, 25-31.

[2]. A.A. Ayi, C.A. Anyama, and V. Khare.J. Mater., 2015, 2015, 7 pages, http://dx.doi.org/10.1155/2015/372716.

[3]. F. Endres, S. Z. El-Abedin. Phys. Chem. Chem. Phys., 2006, 8, 2101-2116.

[4]. S.Z. El-Abedin, F. Endres. Acc. Chem. Res., 2007, 40, 1106-1113.

[5]. W.M. Reichert, J.D. Holbrey, K. B. Vigour, T.D. Morgan, G.A. Broker, R. D. Rogers, Chem. Commun. 2006, 4767-4779.

[6]. A. Taubert, Acta Chim. Slov. 2005, 52, 183186.

[7]. A. Taubert, Z. Li, Dalton Trans., 2007, 7, 723-727.

[8]. T. Nakashima, N. Kimizuka, J. Am. Chem. Soc., 2003, 125, 6386-6387.

[9]. A.A. Ayi, V. Khare, P. Strauch, J. Girard, K. M. Fromm, A. Taubert, Monatsh Chem., 2010 141,1273-1278.

[10]. E.R. Parnham, R.E. Morris, J. Mater. Chem., 2006, 16, 3682-3684.

[11]. E.R Parnham, P.S. Wheatley, R.E. Morris, Chem. Commun., 2006, 380-382. 
[12]. D. Marquardt, Z. Xie, A. Taubert, R. Thomannd and C. Janiak, Dalton Trans., 2011, 40, 8290-8293.

[13]. J. Dupont and J. D. Scholten, Chem. Soc. Rev., 2010, 39, 1780-1804.

[14]. C.S. Consorti, P.A.Z. Suarez, R.F. de Souza, R.A. Burrow, D. H. Farrar, A.J. Lough, W. Loh, L.H. da Silva, J. Dupont J. Phys. Chem. B., 2005, 109, 4341-4349.

[15]. W. Loh, L. H. M. da Silva and J. Dupont, J. Phys. Chem. B, 2005, 109, 4341-4349.

[16]. J. Dupont, J. Braz. Chem. Soc., 2004, 15, 341-350.

[17]. M.-A. Neouze, J. Mater. Chem., 2010, 20, 9593-9607.

[18]. C.B. Murray, C.R. Kagan, M.G. Bawendi, Annu. Rev. Mater. Sci., 2000, 30, 545-610.

[19]. F. Caruso, (ed) Colloids and colloid assemblies. Wiley VCH, Weinheim, 2004.

[20]. S.J. Cavalieri, Y.S. McCarter, S.E. Sharp, J.H. Ortez, I.D. Rankin, R.L. Sautter, R.J. Harbeck, C.A. Spiegel. Manual of antimicrobial susceptibility testing. American Society for microbiology, Washington DC. 2005.

[21]. A. Guinier, X-Ray Diffraction of Crystals, Imperfect Crystals and Amorphous Bodies; Dover: New York, NY, USA, 1994.

[22]. S. Link and M.A. El-Sayed. Annu. Rev. Phys.

Chem., 2003. 54, 331-66.

How to cite this manuscript: Walter Efiok John, Ayi Anyama Ayi*, Chinyere Ayi Anyama, Providence Betelwhobel Ashishie, Bassey Enyi Inah, On The Use of Methylimidazolium Acetate Ionic Liquids as Solvent and Stabilizer in the Synthesis of Cobalt Nanoparticles by Chemical Reduction Method, Adv. I. Chem. A, 2019, 2(2), 175-183.
[23]. U. Kreibig, M. Vollmer, Optical Properties of Metal Clusters. Berlin: Springer, 1995.

[24]. M. Kerker, the Scattering of Light and Other Electromagnetic Radiation. New York: Academic, 1969.

[25]. C.F. Bohren, D.R. Huffman, Absorption and Scattering of Light by Small Particles. New York: Wiley, 1983.

[26]. V. Bansal and S. K. Bhargava, Ionic Liquids as Designer Solvents for the Synthesis of Metal Nanoparticles, In Ionic Liquids: Theory, Properties, New Approaches, Alexander Kokorin (Ed.), InTech, 2011.

[27]. C. Hardacre, J.D, Holbrey, S.E.J. McMath, D.T. Bowron, A.K. Soper, J. Chem. Phys. 2003, 118, 273-278.

[28]. S. Tsuzuki, H. Tokuda, K. Hayamizu, M. Watanabe, J. Phys. Chem. B, 2005, 109, 1647416481

[29]. F. Endres. ChemPhysChem, 2002, 3, 144154.

[30]. J. Dupont, P.A.Z. Suarez. Phys. Chem. Chem. Phys., 2006, 8, 2441-2452.

[31]. M. Antonietti, D. Kuang, B. Smarsly, Y. Zhou. Angew. Chem. Int. Edit, 2004, 43, 49884992.

[32]. Y. Zhou. Current Nanoscience, 2005, 1, 3542. 